\title{
Role of aspiration-induced migration in cooperation
}

\author{
Han-Xin Yang 1 * Zhi-Xi $\mathrm{Wu}^{2}$ 团 and Bing-Hong Wang ${ }^{1,3}$ \\ ${ }^{1}$ Department of Modern Physics, University of Science and Technology of China, Hefei 230026, China \\ ${ }^{2}$ Department of Physics, Umeå University, 90187 Umeå, Sweden \\ ${ }^{3}$ The Research Center for Complex System Science, \\ University of Shanghai for Science and Technology and Shanghai Academy of System Science, Shanghai, 200093 China
}

(Dated: October 30, 2018)

\begin{abstract}
Both cooperation and migration are ubiquitous in human society and animal world. In this paper, we propose an aspiration-induced migration in which individuals will migrate to new sites provided that their payoffs are below some aspiration level. It is found that moderate aspiration level can best favor cooperative behavior. In particular, moderate aspiration level enables cooperator clusters to maintain stably and expand efficiently, whereas induces defector clusters to disintegrate, thus promoting the diffusion of cooperation among population. Our results provide new insights into understanding the role played by migration in the emergence of cooperative behavior.
\end{abstract}

PACS numbers: 87.23.Kg, 02.50.Le, 87.23.Ge, 89.75.Fb

Cooperation is fundamental to biological and social systems. Many important mechanisms have been considered for studying the cooperative behavior, such as costly punishment [1, 2], reputation [3, 4] and social diversity $[5-7]$. As is well-known, migration is a common and essential feature present in animal world and human society. For example, every year millions of animals migrate in the savannas of Africa, and every day thousands of people travel among different countries. Recently, the role of migration has received much attention in the study of evolutionary games 8 - 14$]$.

Migration can be in a random-walk way. For example, Vainstein et al. studied the case in which individuals are located on the sites of a two-dimensional regular lattice and each individual makes an attempt to jump to a nearest neighboring empty site chosen randomly with some probability [10], Meloni et al. consider the case in which individuals are situated on a two-dimensional plane and each individual moves to a randomly chosen position with certain velocity [13]. Besides, the direction of migration can be payoff biased, that is, individuals choose the destination of migration according to payoff. For example, Helbing et al. proposed a success-driven migration mechanism in which individuals will move to the sites with highest estimated payoffs [11], Boyd et al. divided individuals into different subpopulations, the fraction of individuals in subpopulation $i$ who leave and join subpopulation $j$ depends on the payoff difference between two subpopulations [14].

It is noted that in many real-life situations, individuals have to migrate when they cannot find enough resources for living. For example, animals will migrate to other places if they cannot find enough food in the current habitats. Based on such consideration, we propose an

\footnotetext{
*Electronic address: hxyang@mail.ustc.edu.cn

${ }^{\dagger}$ Electronic address: zhi-xi.wu@physics.umu.se

‡Electronic address: bhwang@ustc.edu.cn
}

aspiration-induced migration model, wherein each individual plays with all its neighbors and accumulates payoffs correspondingly; An individual will move to another place if its payoff is lower than the aspiration level, and stay otherwise in the current location. Here the aspiration level can be understood as the minimum living standard for each individual. Considering limited information of the individuals, we assume that, migrants choose new places in a random way.

We use the famous prisoner's dilemma game (PDG) 15 to carry out our researches. In the PDG played by two players, each of whom chooses one of two strategies, cooperation or defection. They both receive payoff $R$ upon mutual cooperation and $P$ upon mutual defection. If one defects while the other cooperates, cooperator receives $S$ while defector gets $T$. The ranking of the four payoff values is: $T>R>P>S$. Following common practice [16], we set $T=b(>1), R=1$, and $P=S=0$, where $b$ represents the temptation to defect.

We assume prisoner's dilemma players on a square with periodic boundary conditions and $L \times L$ sites, which are either empty or occupied by one individual. Initially, an equal percentage of strategies (cooperators or defectors) is randomly distributed among the population. Individuals are updated asynchronously, in a random sequential order. The randomly selected individual plays against individuals sitting on four neighboring nodes (the von Neumann neighborhood), collecting the payoff from the combats. The individual compares its total payoff with its direct neighbors and changes strategy following the one (including itself) with the highest payoff. Before updating strategy, an individual decides whether to stay at or leave its current site. Individual stays in current site if its payoff reaches or exceeds its aspiration level, otherwise it moves to a randomly chosen empty site within its four neighboring sites. To avoid isolated case, we assume that an isolated individual makes mandatory move.

Following previous study [17], the aspiration level $P_{i a}$ for an individual $i$ is defined as $P_{i a}=k_{i} A$, where $k_{i}$ is the number of neighbors of $i$ and $A$ is a control parame- 
ter ( $A$ is the same for all individuals). This definition is based on the following consideration: In real life costless relationships are rare; To maintain social relationship, some cost are required; We assume for simplicity that the needed cost is the same for each link. Thus it is reasonable to assume the aspiration level to be proportional to the number of neighbors.

Figure 1 shows the fraction of cooperators $\rho_{c}$ as a function of the aspiration level $A$ for different values of the temptation to defect $b$ when the fraction of occupied sites $f=0.5$. One can see that, $\rho_{c}$ exhibits discontinuous phase transition with varying $A$ and $\rho_{c}$ is the same between two nearby phase transition points. The value of phase transition point can be determined by the average payoff of an individual (total payoff divided by the number of neighbors), which may be $0,1 / 2, b / 2,1 / 3,2 / 3, b / 3$, $2 b / 3,1, b$ (here we exclude the isolated case in which individual makes mandatory move and four-neighbors case in which individual cannot move). Taking $b=1.5$ as example, the phase transition values of $A$ are $0,1 / 3,1 / 2$, $2 / 3$ respectively (here $0.75,1$ and 1.5 are excluded since $\rho_{c}=0$ for $A>2 / 3$ when $\left.b=1.5\right)$ [18].

From Fig. 1, one can also find, for a fixed value of the temptation to defect $b$, there exists an optimal region of $A$, leading to the highest cooperation level. For $b=1.2$ and $b=1.5$, the optimal region of $A$ is $(0.6,2 / 3]$ and $(0.5,2 / 3]$ respectively, indicating that moderate aspiration level best favors cooperation. For $b=1.51$, the optimal region of $A$ is $(-\infty, 0]$, in which all individuals do not move. This is because, according to the analysis in Ref. [19], compared with never-move case, migration makes it easier for defectors to invade cooperator clusters when $b>1.5$.

How to understand moderate aspiration level best promotes cooperation when $b \leq 1.5$ ? It has been known that in spatial games, cooperators can survive by forming clusters, in which the benefits of mutual cooperation can outweigh losses against defectors, thus enable cooperation to be maintained [16, 20]. For low aspiration level, most individuals do not move. Consequently, cooperatorand defector-clusters coexist and keep almost unchanged in the stationary state, inhibiting the dispersal of cooperation among population. On the contrary, for high aspiration level, most individuals move. Due to the frequent change of neighbors, cooperators can not form clusters to resist the invasion of defectors. As a result, cooperators are doomed to extinct, analogous to the situation arising in the well-mixed population.

For moderate aspiration level, cooperators can form stable clusters since high benefits of mutual cooperation insure them to stay in cooperator clusters, whereas defectors avoid gathering together because the payoffs of mutual defection are low. Figure 2 shows that, during the process of evolution, the number of mobile defectors $N_{m d}$ is much larger than mobile cooperators $N_{m c}$ when $b=1.5, A=0.6$, indicating moderate aspiration level enables cooperator clusters to be sustained whereas induces defector clusters to be disintegrated. A mobile

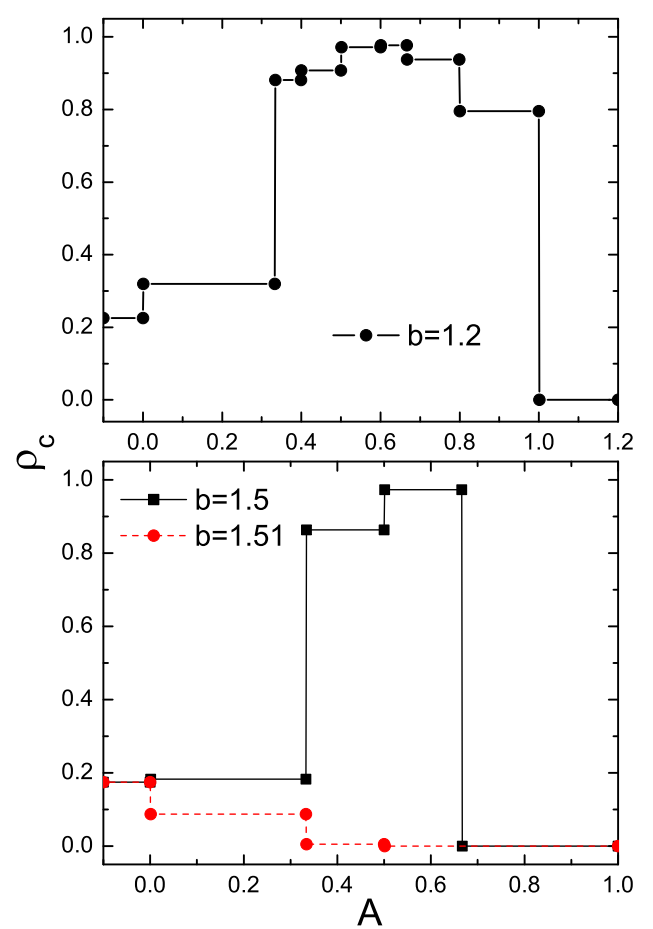

FIG. 1: (Color online) Fraction of cooperators $\rho_{c}$ as a function of the aspiration level $A$ for different values of the temptation to defect $b$. The simulations are for $100 \times 100$ grids with the fraction of occupied sites $f=0.5$. The equilibrium fraction of cooperators results from averaging over 2000 time steps after a transient period of 20000 time steps. Each time step consists of on average one strategy-updating event of all the individuals. Results are averaged over 100 different realizations.

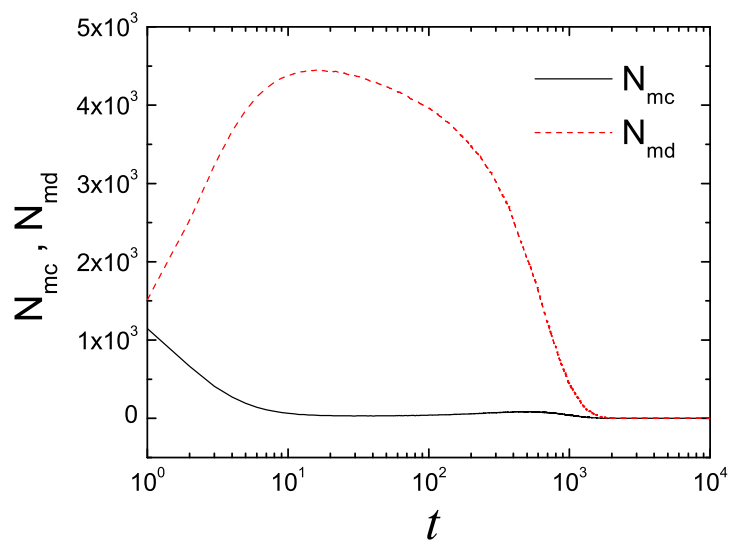

FIG. 2: (Color online) The number of mobile cooperators $N_{m c}$ and mobile defectors $N_{m d}$ as a function of time step $t$ on $100 \times 100$ grids with $50 \%$ empty sites. $b=1.5, A=0.6$. Results are averaged over 100 different realizations. 


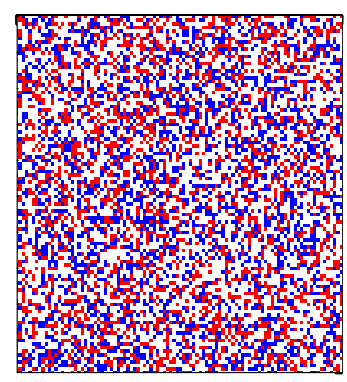

(a)

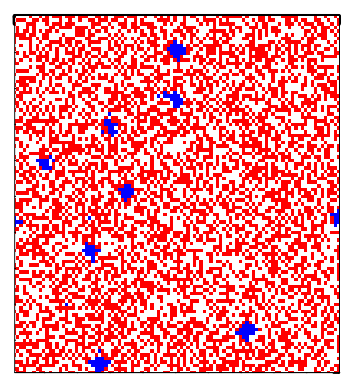

(b)

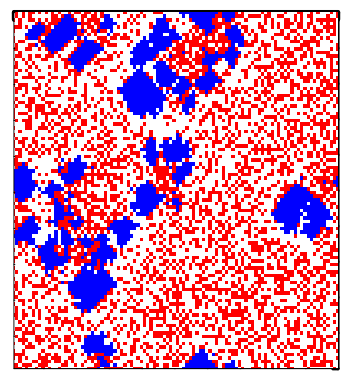

(c)

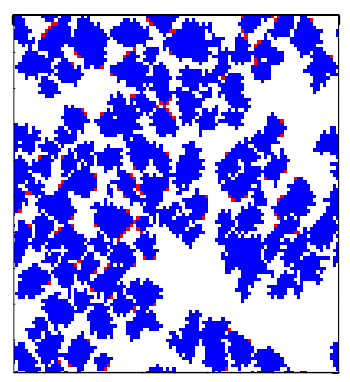

(d)

FIG. 3: (Color online) Snapshots of typical distributions of cooperators (blue) and defectors (red) at different time steps $t$ for $b=1.5$ and $A=0.6$. The simulations are for $100 \times 100$ grids with $50 \%$ empty sites (white). (a) $t=1, \rho_{c}(1)=0.5,(\mathrm{~b}) t=16$, $\rho_{c}(16)=0.0298,(\mathrm{c}) t=370, \rho_{c}(370)=0.302$, and $(\mathrm{d}) t=3000, \rho_{c}(3000)=0.976$.

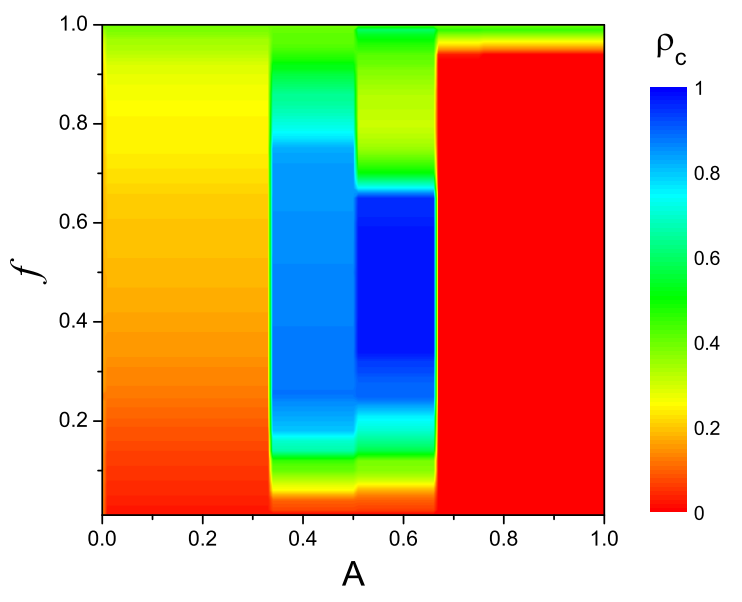

FIG. 4: (Color online) The color code shows the fraction of cooperators $\rho_{c}$ as a function of the aspiration level $A$ and the fraction of occupied sites $f$ on $100 \times 100$ grids. The temptation to defect $b=1.5$. Results are averaged over 100 different realizations.

defector would change to cooperator if it touches cooperator cluster and encounters a cooperator who has the highest payoff among defector and its neighbors (this situation is likely to occur since cooperator clusters usually obtain high payoffs). Thus, for moderate aspiration level, cooperator clusters not only be able to maintain, but also expand due to the existence of migration.

To intuitively understand how moderate aspiration level affects the evolution of cooperation, we plot the distribution of cooperators and defectors on a square lattice at different time steps $t$ for $b=1.5$ and $A=0.6$. Initially $(t=1)$, cooperators and defectors are randomly distributed with the same probability on the square lattice [see Fig. 3(a)]. From Fig. 3(b), we can see that cooperators and defectors are quickly clustered respectively $(t=16)$, and the density of cooperators at this moment is lower than the initial state because cooperators are exposed to much attack of defectors before the formation of steady cooperator clusters. As time step $t$ increases, cooperator clusters expand and defector clusters shrink [see Fig. 3(c)]. Finally, cooperators take over the population and defectors only dispersedly survive nearby cooperator clusters [see Fig. 3(d)], demonstrating that moderate aspiration level can effectively impulse the collapse of defector clusters.

The fraction of occupied sites $f$ also affects the evolution of cooperation. Figure 4 shows the fraction of cooperators $\rho_{c}$ as a function of the aspiration level $A$ and the fraction of occupied sites $f$ together when the temptation to defect $b=1.5$. From Fig. 4, one can see that, the optimal region of $A$ corresponding to the highest cooperation level changes as $f$ varies. For example, the optimal region of $A$ is $(0.5,2 / 3]$ and $(1 / 3,0.5]$ for $f=0.5$ and $f=0.8$ respectively. Besides, one can find that, for a fixed value of $A, \rho_{c}$ varies as $f$ changes. For $A \leq 1 / 3$ and $A>2 / 3, \rho_{c}$ increases as $f$ increases and $f=1$ corresponds to the maximum $\rho_{c}$. For $1 / 3<A \leq 2 / 3$, there exists an intermediate value of $f$, leading to the highest cooperation level.

There is much current interest in studying evolutionary games on various networks [21 27]. It has been found that spatial structure greatly affects the evolution of cooperation [21]. As a natural extension, we now consider 

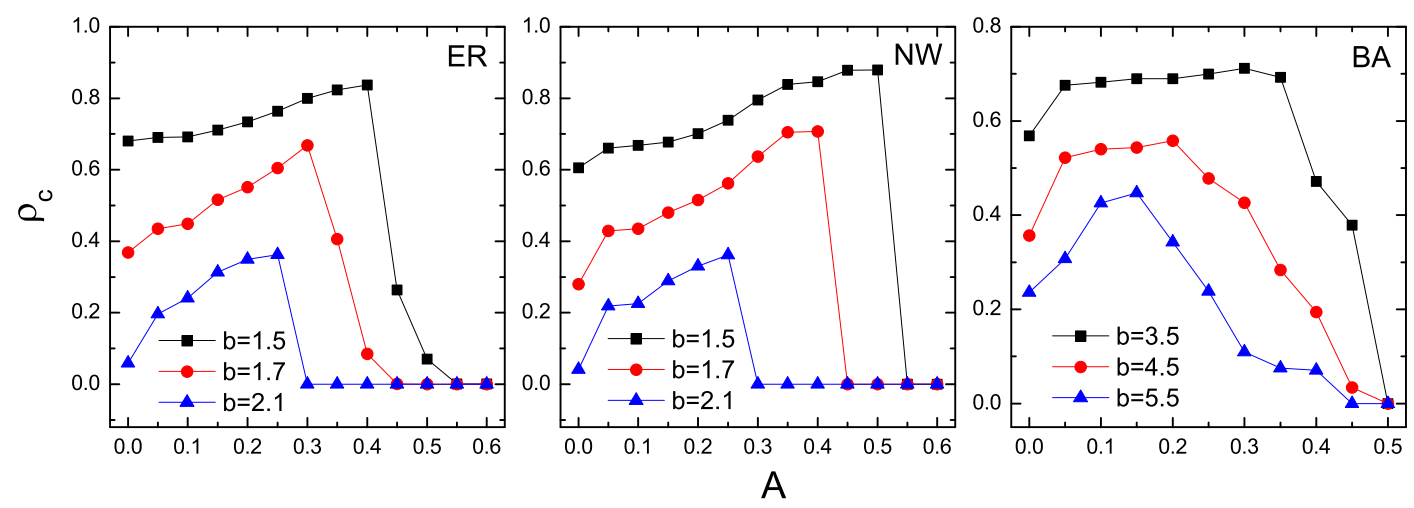

FIG. 5: (Color online) Fraction of cooperators $\rho_{c}$ as a function of the aspiration level $A$ for different values of $b$ when individuals are situated on: ER random graphs (left panel), NW small-world networks (middle panel) and BA scale-free networks (right panel). All networks are of $10^{4}$ nodes with $50 \%$ empty nodes. Average connectivity of ER, NW and BA networks is $10,8,6$ respectively. The equilibrium fraction of cooperators results from averaging over 2000 time steps after a transient period of 20000 time steps. Results are averaged over 100 different realizations.

the aspiration-induced migration on Erdös-Rényi random graphs (ER) 28], Newman-Watts small-world networks (NW) [29] and Barabási-Albert scale-free networks (BA) 30]. In Fig. [5 we report the fraction of cooperators $\rho_{c}$ as a function of the aspiration level $A$ for different values of the temptation to defect $b$. It is interesting to find that, for a fixed value of $b$, there exists an optimal $A$ corresponding to the maximum $\rho_{c}$ for all three networks.

In summary, we have incorporated an aspirationinduced migration mechanism to the evolutionary prisoner's dilemma game. An individual would migrate if its payoff is lower than the aspiration level. We find that, for individuals locating on square lattice and the temptation to defect $b<1.5$, there exists an optimal range of the aspiration level, leading to the maximum cooperation level. We explain such phenomenon by investigating the evolution of cooperator- and defector-clusters. Moderate aspiration level induces cooperator clusters to expand and defector clusters to disintegrate, thus promoting the diffusion of cooperation among population.
We also study the effect of the fraction of occupied sites $f$ on cooperation. Furthermore, studies of aspirationinduced migration model on random graphs, small-world networks and scale-free networks show that, there exists an optimal value of the aspiration level that can best favor cooperative behavior for individuals situated on these networks. Finally we have checked that our conclusions are robust with respect to using different strategy updating rules, such as Fermi updating rule 31, 32] and the finite population analogue of the replicator dynamics 21].

We thank Petter Holme and Wen-Xu Wang for useful discussions. This work is funded by the National Basic Research Program of China (973 Program No. 2006CB705500), the National Natural Science Foundation of China (Grant Nos. 10975126, 10635040), the Specialized Research Fund for the Doctoral Program of Higher Education of China (Grant No. 20093402110032). Z.X.W. acknowledges the support from the Swedish Research Council.
[1] C. Hauert, A. Traulsen, H. Brandt, M. A. Nowak, and K. Sigmund, Science 316, 1905 (2007).

[2] H. Ohtsuki, Y. Iwasa, and M. A. Nowak, Nature 457, 79 (2009).

[3] M. A. Nowak and K. Sigmund, Nature 393, 573 (1998).

[4] H. Ohtsuki and Y. Iwasa, J. Theor. Biol. 231, 107 (2004).

[5] F. C. Santos, M. D. Santos, and J. M. Pacheco, Nature 454, 213 (2008).

[6] M. Perc and A. Szolnoki, Phys. Rev. E 77, 011904 (2008).

[7] H.-X. Yang, W.-X. Wang, Z.-X. Wu, Y.-C. Lai, and B.-H. Wang, Phys. Rev. E 79, 056107 (2009).
[8] M. Enquist and O. Leimar, Anim. Behav. 45, 747 (1993).

[9] C. A. Aktipis, J. Theor. Biol. 231, 249 (2004).

[10] M. H. Vainstein, A. T. C. Silva, and J. J. Arenzon, J. Theor. Biol. 244, 722 (2007).

[11] D. Helbing and W. Yu, Proc. Natl. Acad. Sci. USA 106, 3680 (2009).

[12] E. A. Sicardi, H. Fort, M. H. Vainstein, and J. J. Arenzon, J. Theor. Biol. 256, 240 (2009).

[13] S. Meloni, A. Buscarino, L. Fortuna, M. Frasca, J. Gómez-Gardenẽs, V. Latora, and Y. Moreno, Phys. Rev. E 79, 067101 (2009). 
[14] R. Boyd and P. J. Richerson, J. Theor. Biol. 257, 331 (2009).

[15] R. Axelrod and W. D. Hamilton, Science 211, 1390 (1981).

[16] M. A. Nowak and R. M. May, Nature 359, 826 (1992).

[17] X. Chen and L. Wang, Phys. Rev. E 77, 017103 (2008).

[18] Note that the phase transition points disappear when $f \simeq 1$.

[19] D. Helbing and W. Yu, Adv Complex Syst 11, 641 (2008).

[20] J. Gómez-Gardenẽs, M. Campillo, L. M. Floría, and Y. Moreno, Phys. Rev. Lett. 98, 108103 (2007).

[21] F. C. Santos and J. M. Pacheco, Phys. Rev. Lett. 95, 098104 (2005).

[22] H. Ohtsuki, C. Hauert, E. Lieberman, and M. A. Nowak, Nature 441, 502 (2006).

[23] J. Ren, W.-X. Wang, and F. Qi, Phys. Rev. E 75, 045101
(R) (2007).

[24] Z. Rong, X. Li, and X. Wang, Phys. Rev. E 76, 027101 (2007).

[25] F. Fu, T. Wu, and L. Wang, Phys. Rev. E 79, 036101 (2009).

[26] Z.-X. Wu and P. Holme, Phys. Rev. E 80, 026108 (2009).

[27] A. Szolnoki, M. Perc, and G. Szabó, Phys. Rev. E 80, 056104 (2009).

[28] P. Erdős and A. Rényi, Publ. Math. (Debrecen) 6, 290 (1959).

[29] M. E. J. Newman and D. J. Watts, Phys. Lett. A 263 , 341 (1999).

[30] A.-L. Barabasi and R. Albert, Science 286, 509 (1999).

[31] G. Szabó and C. Tőke, Phys. Rev. E 58, 69 (1998).

[32] A. Traulsen, J. M. Pacheco, and M. A. Nowak, J. Theor. Biol. 246, 522 (2007). 